\title{
Biventricular structural and functional responses to aortic constriction in a rabbit model of chronic right ventricular pressure overload
}

\author{
Christian Apitz, MD, ${ }^{\mathrm{a}, \mathrm{c}}$ Osami Honjo, MD, ${ }^{\mathrm{b}}$ Tilman Humpl, MD, ${ }^{\mathrm{a}}$ Jing Li, MD, ${ }^{\mathrm{a}}$ Renato S. Assad, MD, ${ }^{\mathrm{d}}$ \\ Mi Y. Cho, MD, ${ }^{a}$ James Hong, MD, ${ }^{a}$ Mark K. Friedberg, MD, ${ }^{a}$ and Andrew N. Redington, MD $^{\mathrm{a}}$
}

\begin{abstract}
Objectives: Chronic right ventricular (RV) pressure overload results in pathologic RV hypertrophy and diminished RV function. Although aortic constriction has been shown to improve systolic function in acute RV failure, its effect on RV responses to chronic pressure overload is unknown.
\end{abstract}

\begin{abstract}
Methods: Adjustable vascular banding devices were placed on the main pulmonary artery and descending aorta. In 5 animals (sham group), neither band was inflated. In 9 animals (PAB group), only the pulmonary arterial band was inflated, with adjustments on a weekly basis to generate systemic or suprasystemic RV pressure at 28 days. In 9 animals, both pulmonary arterial and aortic devices were inflated (PAB $+\mathrm{AO}$ group), the pulmonary arterial band as for the PAB group and the aortic band adjusted to increase proximal systolic blood pressure by approximately $20 \mathrm{~mm} \mathrm{Hg}$. Effects on the functional performance were assessed 5 weeks after surgery by conductance catheters, followed by histologic and molecular assessment.
\end{abstract}

Results: Contractile performance was significantly improved in the $\mathrm{PAB}+\mathrm{AO}$ group versus the $\mathrm{PAB}$ group for both ventricles. Relative to sham-operated animals, both banding groups showed significant differences in myocardial histologic and molecular responses. Relative to the $\mathrm{PAB}$ group, the $\mathrm{PAB}+\mathrm{AO}$ group showed significantly decreased RV cardiomyocyte diameter, decreased RV collagen content, and reduced RV expression of endothelin receptor type $\mathrm{B}$, matrix metalloproteinase 9 , and transforming growth factor $\beta$ genes.

Conclusions: Aortic constriction in an experimental model of chronic RV pressure overload not only resulted in improved biventricular systolic function but also improved myocardial remodeling. These data suggest that chronically increased left ventricular afterload leads to a more physiologically hypertrophic response in the pressure-overloaded RV. (J Thorac Cardiovasc Surg 2012;144:1494-501)

The right ventricle (RV) may be subjected to an abnormally high afterload in patients with various types of congenital and acquired heart disease and also in idiopathic, hereditary or other forms of pulmonary arterial (PA) hypertension. ${ }^{1}$ The RV typically shows progressive remodeling, including increased wall thickness, myocardial hypertrophy, and interstitial fibrosis. This initially adaptive compensatory hypertrophy eventually becomes maladaptive and may eventually progress to RV dilatation and failure, ${ }^{2,3}$ the development of which is a universally poor prognostic feature. ${ }^{4}$ Maladaptive hypertrophy and interstitial fibrosis

From the Divisions of Cardiology ${ }^{\mathrm{a}}$ and Cardiac Surgery, ${ }^{\mathrm{b}}$ Hospital for Sick Children, University of Toronto, Ontario, Canada; the Pediatric Heart Centre, ${ }^{c}$ University Children's Hospital, Giessen, Germany; and the Heart Institute, ${ }^{\mathrm{d}}$ University of São Paulo, São Paulo, Brazil.

Supported by a research scholarship of the Deutsche Herzstiftung e.V., Frankfurt, Germany (to C.A.).

Disclosures: Authors have nothing to disclose with regard to commercial support.

M.K.F. and A.N.R. are equal principal authors.

Received for publication March 19, 2012; revisions received May 9, 2012; accepted for publication June 12, 2012; available ahead of print July 23, 2012.

Address for reprints: Andrew N. Redington, MD, Division of Cardiology, The Hospital for Sick Children, University of Toronto, 555 University Ave, Toronto, Ontario, Canada M5G 1X8 (E-mail: andrew.redington@sickkids.ca).

$0022-5223 / \$ 36.00$

Copyright (c) 2012 by The American Association for Thoracic Surgery http://dx.doi.org/10.1016/j.jtcvs.2012.06.027 are usually associated with marked changes in myocardial fibrotic signaling. Among others increased levels of endothelin 1 (ET-1), matrix metalloproteinase (MMP) 9, and transforming growth factor $\beta$ (TGF- $\beta$ ) have been observed as markers of maladaptive hypertrophy and fibrosis in models of left ventricular (LV) dysfunction and failure and may also play a role in $\mathrm{RV}$ failure. ${ }^{5}$

The pathophysiology of RV failure, particularly with regard to adverse ventricular-ventricular interactions, is becoming increasingly understood. For example, in a study of patients with chronic idiopathic pulmonary hypertension, right heart dilation altered LV geometry and diastolic function, as assessed by Doppler echocardiography, and was associated with worse outcomes. ${ }^{6}$ Furthermore, in a magnetic resonance study, Gan and coworkers ${ }^{7}$ demonstrated that cardiac output was inversely related to $\mathrm{LV}$ end-diastolic dimension (because it is compressed by the $\mathrm{RV}$ ), rather than to RV function per se. ${ }^{7}$ Conversely, it may therefore be possible that modification of these adverse ventricular-ventricular interactions may lead to some benefits. It is well known that superficial myocardial fibers are shared and continuous between the RV and the $\mathrm{LV}$, providing an anatomic basis for normal and abnormal ventricular-ventricular interactions. ${ }^{8}$ Indeed, in an elegant 


$$
\begin{array}{ll}
\text { Abbreviations and Acronyms } \\
C T G F=\text { connective tissue growth factor gene } \\
E D N 1=\text { endothelin } 1 \text { gene } \\
E D N R A=\text { endothelin receptor type A gene } \\
E D N R B=\text { endothelin receptor type B gene } \\
\text { EDPVR }=\text { end-diastolic pressure-volume } \\
\text { relationship } \\
\text { ESPVR }=\text { end-systolic pressure-volume } \\
& \text { relationship } \\
\text { ET- } 1= & \text { endothelin } 1 \\
\text { GAPDH } & \text { glyceraldehyde } 3 \text {-phosphate } \\
& \text { dehydrogenase gene } \\
\text { LV } & =\text { left ventricle } \\
\text { MMP }= & \text { matrix metalloproteinases } \\
M M P 2 & =\text { matrix metalloproteinase } 2 \text { gene } \\
M M P 9= & \text { matrix metalloproteinase } 9 \text { gene } \\
\text { MT } & =\text { Masson trichrome } \\
\text { PA } & =\text { pulmonary artery } \\
\text { PRSW } & =\text { preload recruitable stroke work } \\
\text { PSR } & =\text { picrosirius red } \\
\text { RV } & =\text { right ventricle } \\
\text { TGF- } \beta= & \text { transforming growth factor } \beta
\end{array}
$$

study of normal hearts, in which the ventricles were electrically isolated but mechanically intact, Damiano and colleagues ${ }^{9}$ showed that under basal conditions $\mathrm{LV}$ contraction contributed more than $65 \%$ of the work of the normal RV. These normal interactions form the basis of beneficial interventions in models of acute RV failure. Yamashita and coworkers ${ }^{10}$ showed that increased LV afterload, produced by aortic constriction, both restored LV dimensions and improved RV stroke volume in the setting of acute pulmonary embolic shock. Similarly, Belenkie and coworkers ${ }^{11}$ showed a beneficial effect of aortic constriction (independent of changes in coronary flow) in experimental RV failure induced by acute banding of the PA, ${ }^{11}$ and our own recent data in a similar PAbanding model showed that improvement in RV contractility assessed by conductance catheter could be induced both by acute aortic banding and by pharmacologically increasing LV afterload with norepinephrine. ${ }^{12}$ We speculated that the improved RV contractility was manifested through harnessing of the Anrep effect of increased LV afterload, leading to increased LV contractility, which in turn was transmitted to the RV through the crosstalk mechanism shown by Damiano and colleagues, ${ }^{9}$ but clearly other factors, such as changes in septal position, may also contribute to this phenomenon.

To date, there have been no studies of the effect of increased $L V$ afterload on $R V$ responses in chronic $R V$ failure. The purpose of this study was therefore to investigate the effect of chronic aortic constriction on biventricular structural and functional responses in an experimental model of chronic RV pressure overload. We hypothesized that aortic constriction would improve RV function and myocardial remodeling in this setting.

\section{MATERIALS AND METHODS}

All authors had full access to and take full responsibility for the integrity of the data. All authors have read and agree to the article as written. All experiments were approved by the animal ethics committee of the Hospital for Sick Children, Toronto, Ontario, Canada, and were done in accordance with the "Guiding Principles in the Care and Use of Animals" of the American Physiologic Society.

\section{Preparation}

Twenty-three adult New Zealand white rabbits were studied. After intravenous cannulation (24G Angiocath; BD, Franklin Lakes, NJ) of the ear marginal vein, anesthesia was initiated by the use of isoflurane $(3 \%)$ and acepromazine maleate (INN acepromazine, $1.1 \mathrm{mg} / \mathrm{kg}$ ). After tracheal intubation, ventilation was controlled mechanically to maintain normal blood gas values $\left(\mathrm{PaCO}_{2}\right.$ of $\left.32-35 \mathrm{~mm} \mathrm{Hg}\right)$. General anesthesia was maintained with isoflurane $(1.5 \%-2 \%)$. Heart rate and oxygen saturation were continuously monitored.

\section{Banding Devices}

For incremental banding of the pulmonary trunk and ascending aorta, we used a recently developed adjustable banding device (ABS; Silimed Inc, Rio de Janeiro, Brazil). ${ }^{13}$ The band consists of a banding ring, a connecting tube, and an inflation reservoir. The banding ring is a $\mathrm{C}$-shaped hydraulic cuff with a $5 \mathrm{~mm}$ width and a rigid outer layer, reinforced with a polyester mesh, which keeps it from deforming centrifugally. The cuff compresses the lumen of the vessel when expanded in proportion to the volume injected into the inflation reservoir. The connecting tube hermetically connects the banding ring to the inflation reservoir, which is covered by a silicone membrane to allow repeated percutaneous needle puncture for adjustment of the degree of vascular constriction. In all animals, adjustable banding devices were placed on the pulmonary trunk and descending aorta through a left lateral thoracotomy, then the connecting tubes were tunneled toward each of the inflation reservoirs, which were placed subcutaneously in the neck.

After band placement, the animals were then allocated to either the PAB group, which underwent PA band inflation alone $(n=9)$; the PAB $+A O$ group, which underwent both PA and aortic band inflation $(n=9)$; or the sham group, which did not have the devices inflated $(n=5)$.

After a recovery period of 7 days, each animal in the $\mathrm{PAB}$ and $\mathrm{PAB}+\mathrm{AO}$ groups received stepwise percutaneous inflation of the cuff around the PA to induce RV pressure overload. Doppler echocardiography was used to monitor RV pressure (estimated by Doppler assessment of the tricuspid regurgitation) and the RV/PA pressure gradient (estimated by Doppler assessment of gradient across the PA band). Three incremental PA band adjustments were performed on a weekly basis. Initially, the banding was calibrated to an RV pressure of approximately half systemic pressure, at second banding to between two thirds and three quarters of systemic pressure, and to systemic levels on the $3 \mathrm{rd}$ occasion. In the $\mathrm{PAB}+\mathrm{AO}$ group, the aortic banding was also adjusted at each stage to maintain a gradient of approximately $20 \mathrm{~mm} \mathrm{Hg}$ across the band (as measured by Doppler echocardiography).

\section{Functional Assessment}

Hemodynamic measurements were performed in all animals 5 weeks after pulmonary banding-operation (after 2 weeks of systemic RV pressure) A $3 \mathrm{~F}$ conductance catheter (Millar Instruments, Inc, Houston, Tex) was advanced to each ventricle for simultaneous measurement of pressures and 
volumes through a neck vein and a neck artery, respectively. For preload reduction, required to obtain pressure-volume relationships, a balloon catheter (10-mm Tyshak balloon; NuMED Canada Inc, Cornwall, Ontario, Canada) was introduced through a groin vein and was placed in the inferior caval vein under fluoroscopic guidance. For all conditions, steady-state hemodynamic data were recorded during short periods of suspended ventilation at end-expiration. Indices of systolic and diastolic function were derived from pressure-volume loops recorded during inflation of a balloon catheter in the inferior caval vein to reduce RV preload. For systolic ventricular function, we determined end-systolic elastance as the slope of the end-systolic pressure-volume relationship (ESPVR) and preload recruitable stroke work (PRSW) from a family of pressure-volume loops during progressive occlusion of the inferior caval vein. Diastolic stiffness was determined as the slope of the end-diastolic pressure-volume relationship (EDPVR).

\section{Tissue Collection}

Each animal was killed after hemodynamic measurements were made. The LV and RV samples were cut into 2 parts, 1 of which was immediately snap-frozen in liquid nitrogen and stored at $-80^{\circ} \mathrm{C}$ for RNA and protein analysis. The other part was fixed in $10 \%$ neutral-buffered formaldehyde and embedded in paraffin. The 5- $\mu \mathrm{m}$ cross-sections of RV and LV were cut and stained with hematoxylin-eosin, picrosirius red (PSR) F3BA, and Masson trichrome (MT) stains.

\section{Histologic Analysis}

Interstitial collagen in transverse cardiac section was evaluated by PSR and MT staining. Interstitial collagen was identified in the PSR-stained sections by its red appearance and in the MT-stained sections by its blue appearance. The cardiac interstitial collagen content was measured and expressed as a percentage of the total collagenous and noncollagenous areas in the entire visual field of the section by automated planimetry with Adobe Photoshop CS2 software (Adobe Systems Inc, San Jose, Calif). The myocyte diameter was measured with images captured from hematoxylin-eosin-stained sections. A point-to-point perpendicular line was placed across the longitudinally cut myocyte at the level of the nucleus, and this diameter length was then measured by the National Institutes of Health ImageJ analysis program (http://rsbweb.nih.gov/ij/). All the longitudinally directed myocytes with a distinct cell border (at the level of the nucleus) within the sampling field were measured and averaged to provide the mean cardiomyocyte diameter. Transverse or obliquely cut myocytes were excluded. Cell width was determined from longitudinally positioned myocytes to reduce the error of determining such for myocytes that might not have been cut precisely perpendicular to their long axis.

\section{Real-Time Reverse Transcriptase-Polymerase Chain Reaction Analysis}

Total RNA was extracted from RV and LV tissues with TRIzol Reagent (Invitrogen Corp, Grand Island, NY). Reverse transcription proceeded with $1 \mu \mathrm{g}$ of total RNA with SuperScript III First-Strand Synthesis System (Invitrogen). Real-time polymerase chain reaction was carried out with SYBR Green Master Mix (Applied Biosystems, Life Technologies Corporation, Carlsbad, Calif). Glyceraldehyde 3-phosphate dehydrogenase gene $(G A P D H)$ was used as the endogenous reference. Primers are listed as follows: ET-1 gene (EDN1), forward 5' -ACTTCTGCCACCTGGACATCA- $3^{\prime}$, reverse 5'-ACGCTGCCCTGGTAGGAAAT-3'; endothelin receptor type A gene (EDNRA), forward 5'-GCTTCTTGCTGCTCATGGATTAC-3', reverse $5^{\prime}$ - CCGAGGTCATCAGGCTCTTG-3'; endothelin receptor type B gene $(E D N R B)$, forward $5^{\prime}$-CTGGCCATTTGGAGCTGAGA- $3^{\prime}$, reverse 5'-TTTGGAACCCCAATTCCTTTAA-3'; MMP-2 gene (MMP2), forward 5'- AGGACTACGACCGCGACAAG-3', reverse 5'- TGTTGCCCAG GAAGGTGAAG -3'; MMP-9 gene (MMP9), forward 5'-CTTCCAACTTT GACAGCGACA- $3^{\prime}$, reverse $5^{\prime}$-GGAGTGATCCAAGCCCAGTG- ${ }^{\prime}$; TGF- $\beta$ gene $(T G F B)$, forward 5'-AGGGCTACCACGCCAACTT-3', reverse: $5^{\prime}$-CCGGGTTGTGCTGGTTGTAC- $3^{\prime}$, connective tissue growth factor gene $(C T G F)$, forward $5^{\prime}$-CCCTGCGTCTTCGGTGGC-3', reverse $5^{\prime}$-AGGCAGTTGGCTCGCATCAT-3'; and GAPDH, reverse $5^{\prime}$-AGG CCGTGGGCAAGGT- ${ }^{\prime}$, reverse $5^{\prime}$-CCTCGGATGCCTGCTTCA-3'.

\section{Statistical Analysis}

Data are expressed as mean \pm SEM. Results were analyzed by analysis of variance for repeated measurements. GraphPad software (GraphPad Software Inc, San Diego, Calif) was used for statistical analysis.

\section{RESULTS}

\section{Hemodynamic and Functional Characteristics}

During the postsurgical periods, all animals gained weight and showed no clinical signs of heart failure. Water and food were available ad libitum, and intake was normal. Populations were similar in each group in terms of age and body weight at time of the initial and terminal experiments. Hemodynamic and functional data are listed in Table 1. As expected, PA banding resulted in a significant increase in RV systolic pressure relative to the sham group $(P<.01)$ and was not different between the PAB and PAB $+\mathrm{AO}$ groups. The slopes of RV ESPVR and RV PRSW were also significantly increased in

TABLE 1. Hemodynamic and functional data

\begin{tabular}{|c|c|c|c|c|c|c|}
\hline & Sham & PAB & $\begin{array}{c}P \\
\text { value* }^{*} \\
\end{array}$ & $\mathbf{P A B}+\mathbf{A O}$ & $\begin{array}{c}P \\
\text { value } \dagger\end{array}$ & $\begin{array}{c}P \\
\text { value } \neq \\
\end{array}$ \\
\hline RV Pes (mm Hg) & $14.4 \pm 1.2$ & $52.6 \pm 3.1$ & $<.01$ & $52.7 \pm 2.3$ & $<.01$ & NS \\
\hline RV Ped (mm Hg) & $1.6 \pm 0.4$ & $2.1 \pm 0.4$ & NS & $4.0 \pm 0.7$ & $<.01$ & $<.05$ \\
\hline RV PRSW (mm Hg) & $1.5 \pm 1.3$ & $4.1 \pm 0.9$ & $<.05$ & $13.6 \pm 5.1$ & $<.0001$ & $<.05$ \\
\hline RV ESPVR (mm Hg/mL) & $2.7 \pm 0.3$ & $7.7 \pm 2.9$ & $<.05$ & $13.9 \pm 4.9$ & $<.0001$ & $<.05$ \\
\hline $\mathrm{RV} \operatorname{EDPVR}(\mathrm{mm} \mathrm{Hg} / \mathrm{mL})$ & $0.8 \pm 0.3$ & $1.3 \pm 0.5$ & NS & $2.2 \pm 0.5$ & NS & NS \\
\hline LV Pes (mm Hg) & $36.1 \pm 2.2$ & $48.0 \pm 0.9$ & NS & $74.3 \pm 3.9$ & $<.01$ & $<.05$ \\
\hline LV Ped (mm Hg) & $1.2 \pm 0.5$ & $1.5 \pm 0.5$ & NS & $4.9 \pm 0.6$ & $<.01$ & $<.01$ \\
\hline LV PRSW (mm Hg) & $13.3 \pm 5.7$ & $13.4 \pm 1.2$ & NS & $22.6 \pm 4.6$ & $<.05$ & $<.05$ \\
\hline LV ESPVR (mm Hg/mL) & $10.6 \pm 0.9$ & $11.3 \pm 2.5$ & NS & $18.2 \pm 1.5$ & $<.05$ & $<.05$ \\
\hline LV EDPVR $(\mathrm{mm} \mathrm{Hg} / \mathrm{mL})$ & $1.1 \pm 0.1$ & $1.3 \pm 0.3$ & NS & $2.4 \pm 0.4$ & $<.05$ & $<.05$ \\
\hline
\end{tabular}

All values are mean \pm SEM. EDPVR, End-diastolic pressure-volume relationship; ESPVR, end-systolic pressure-volume relationship; $L V$, left ventricular; $N S$, not significant $(P>.05) ; P A B$, pulmonary arterial banding alone [group]; $P A B+A O$, pulmonary arterial and aortic banding [group]; Pes, end-systolic pressure; $P e d$, end-diastolic pressure; $P R S W$, preload recruitable stroke work; $R V$, right ventricular. $*$ Sham versus pulmonary arterial banding alone. $\dagger$ Sham versus pulmonary arterial banding plus aortic banding.

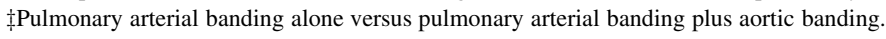



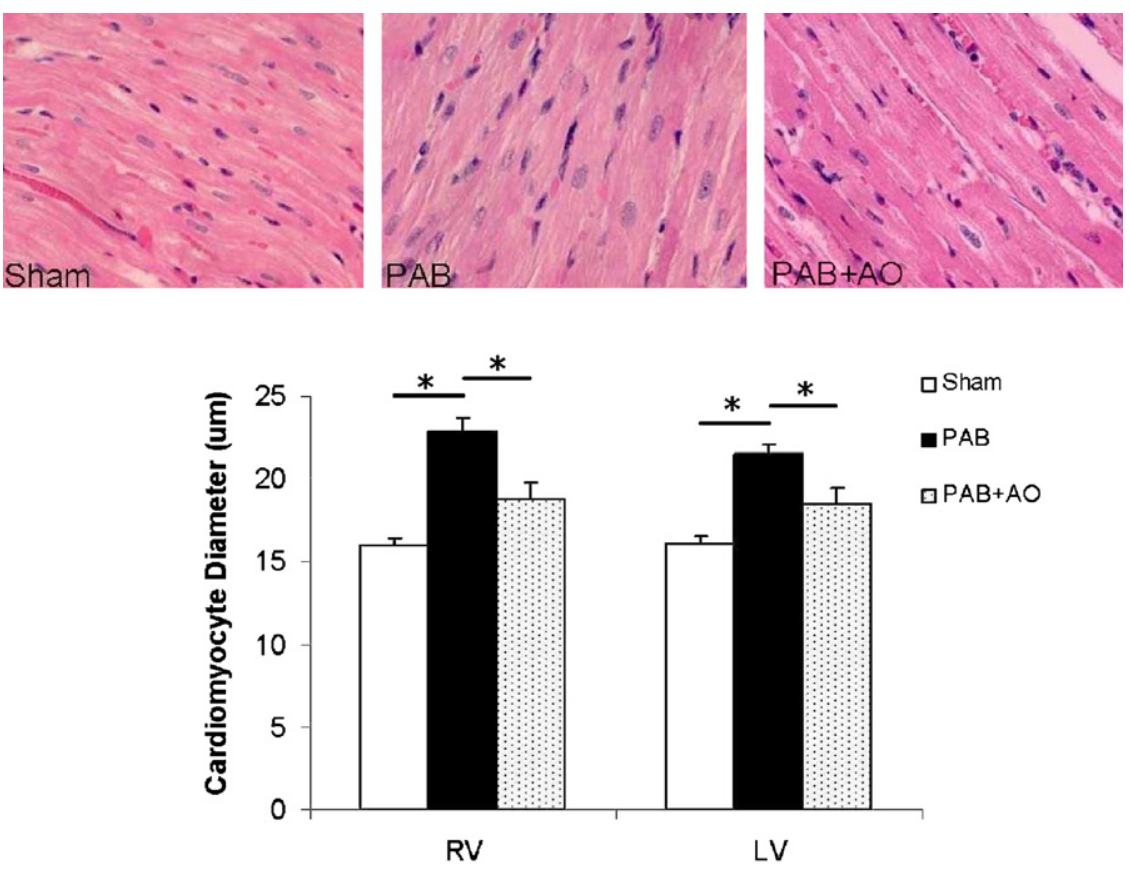

FIGURE 1. Hematoxylin-eosin staining showed a significantly increased cardiomyocyte diameter in the pulmonary arterial banding only $(P A B)$ group for the right ventricle $(R V)$ and for the left ventricle $(L V)$. Addition of aortic banding $(P A B+A O)$ resulted in a significantly decreased cardiomyocyte diameter for both ventricles. Asterisk indicates $P<.05$.

the PAB group relative to the sham group. End-diastolic pressure in the RV tended to increase in the PAB group relative to the sham group, but not significantly. In the LV, end-systolic pressure, end-diastolic pressure, and the slopes of LV ESPVR and LV PRSW did not differ significantly between the PAB group and the sham group.

Additional aortic banding $(\mathrm{PAB}+\mathrm{AO})$ resulted in further functional improvement. Relative to the $\mathrm{PAB}$ group, ESPVR and PRSW were significantly higher in the $\mathrm{PAB}+\mathrm{AO}$ group for the RV (RV ESPVR, 13.9 vs $7.7 \mathrm{~mm}$ $\mathrm{Hg} / \mathrm{mL} ; P<.05 ; \mathrm{RV}$ PRSW, 13.6 vs $4.1 ; P<.05)$ and for the LV (LV ESPVR 18.2 vs $11.3 \mathrm{~mm} \mathrm{Hg} / \mathrm{mL} ; P<.05$; LV PRSW, 22.6 vs $13.4 ; P<.05$ ), reflecting improved biventricular contractility.

Diastolic RV stiffness, as assessed by RV EDPVR, was not different in either intervention group relative to the sham group, whereas the end-diastolic RV pressure was significantly increased in the $\mathrm{PAB}+\mathrm{AO}$ group relative to the $\mathrm{PAB}$ and sham groups. In the LV, there were significant increases in both LV EDPVR and end-diastolic LV pressure in the $\mathrm{PAB}+\mathrm{AO}$ group relative to the $\mathrm{PAB}$ and sham groups.

\section{Histologic Remodeling}

Hematoxylin-eosin staining showed significantly increased cardiomyocyte diameters in the PAB group relative to the sham group $(22.88 \pm 0.87 \mu \mathrm{m}$ vs $16.03 \pm 0.47 \mu \mathrm{m}$ for the $\mathrm{RV}$ and $21.53 \pm 0.54 \mu \mathrm{m}$ vs $16.12 \pm 0.41 \mu \mathrm{m}$ for the LV; Figure 1). Additional aortic banding resulted in significantly decreased cardiomyocyte diameters in the $\mathrm{PAB}+\mathrm{AO}$ group $(18.8 \pm 0.99 \mu \mathrm{m}$ for the $\mathrm{RV}$ and $18.47 \pm$ $1.01 \mu \mathrm{m}$ for the $\mathrm{LV}$ ) relative to the PAB group.

Both PSR and MT staining revealed significant increases in collagen in both the RV (PSR staining, 10.78\% $\pm 1.39 \%$ vs $3.74 \% \pm 0.34 \%$; MT staining, $9.2 \% \pm 1.73 \%$ vs $2.12 \%$ $\pm 0.31 \%$ ) and the LV (PSR staining, $5.28 \% \pm 0.48 \%$ vs $2.86 \% \pm 0.3 \%$; MT staining, $2.5 \% \pm 0.46 \%$ vs $0.9 \% \pm$ $0.23 \%$ ) for the PAB group relative to the sham group $(P<.05)$. Addition of aortic banding resulted in significant decreases in collagen in both the RV (PSR staining, 6.61\% $\pm 0.85 \%$; MT staining, $4.78 \% \pm 0.77 \%$ ) and the LV (PSR staining, $3.81 \% \pm 0.36 \%$; MT staining, $1.8 \% \pm 0.33 \%)$ for the $\mathrm{PAB}+\mathrm{AO}$ group $(P<.05)$ (Figure 2$)$.

\section{Molecular Assessment}

Expression of EDN1 was significantly increased in the RV myocardium in both the PAB group $(2.22 \pm 0.42)$ and the $\mathrm{PAB}+\mathrm{AO}$ group $(2.18 \pm 0.28)$ relative to the sham group $(1.07 \pm 0.19 ; P<.05$; Figure $3, A)$. Interestingly, expression of $E D N 1$ was also significantly increased in the LV myocardium in both the PAB group $(2.5 \pm 0.26)$ and the $\mathrm{PAB}+\mathrm{AO}$ group $(2.99 \pm 0.19)$ relative to the sham group $(1.14 \pm 0.26 ; P<.05$; Figure $4, A)$. RV and LV myocardial expressions of EDNRA were not different in either intervention group relative to the sham group (RV, $1.16 \pm 0.11$ and $1.06 \pm 0.07$ vs $1.02 \pm 0.11$, respectively; $P$ not significant), $(\mathrm{LV}, 1.1 \pm 0.06$ and $0.89 \pm 0.07$ vs $1.02 \pm 0.11$, 

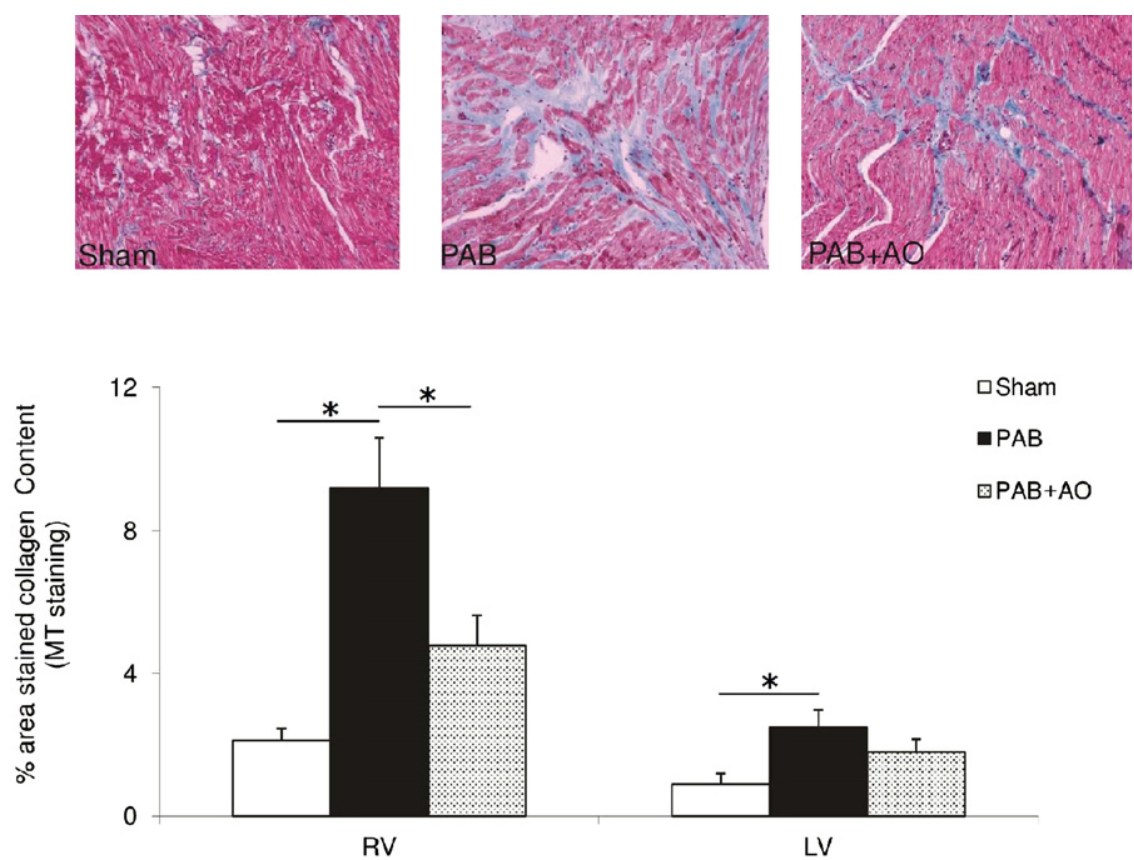

FIGURE 2. Representative sections showing Masson trichrome (MT) staining for collagen content. The bar graph of the quantitative analysis shows increased collagen in response to pulmonary arterial banding $(P A B)$ in both the right ventricle $(R V)$ and the left ventricle $(L V)$. Addition of aortic banding $(P A B+A O)$ significantly attenuated this increase in the right ventricle but not in the left ventricle. Asterisk indicates $P<.05$.

respectively; $P$ not significant). EDNRB expression, in contrast was significantly elevated in the RV in the PAB group $(1.95 \pm 0.3)$ relative to the sham group $(1.02 \pm 0.1 ; P<.05)$ but not in the $\mathrm{PAB}+\mathrm{AO}$ group $(1.52 \pm 0.3 ; P$ not significant; Figure $3, A$ ). There were no differences among the groups in $E D N R B$ expression in the LV (Figure 4, $A$ ).

$M M P 9$ expressions were significantly elevated in the RV myocardium in both the PAB group $(2.12 \pm 0.32)$ and the $\mathrm{PAB}+\mathrm{AO}$ group $(1.38 \pm 0.12)$ relative to the sham group $(0.88 \pm 0.2 ; P<.05)$, whereas in the LV myocardium $M M P 9$ expressions were not different in either the $\mathrm{PAB}$ group $(2.32 \pm 0.39)$ or the $\mathrm{PAB}+\mathrm{AO}$ group $(1.83 \pm 0.18)$ relative to the sham group $(1.21 \pm 0.43 ; P$ not significant $)$. $M M P 2$ expressions were not elevated $(1.07 \pm 0.16$ and 0.96 \pm 0.13 vs $1.01 \pm 0.16$ ) in either ventricle. In the RV myocardium, TGF- $\beta$ expression was significantly increased in the PAB group $(1.4 \pm 0.13)$ relative to the sham group $(0.84 \pm 0.16 ; P<.05$; Figure $3, B)$ and was significantly lower in the $\mathrm{PAB}+\mathrm{AO}$-group $(0.93 \pm 0.1)$ relative to the PAB group $(P<.05)$. A similar pattern of TGF- $\beta$ expression was seen in the LV (Figure $4, B$ ). There were no statistically significant differences in $C T G F$ expressions between any of the groups in either ventricle.

\section{DISCUSSION}

This study demonstrates that chronic aortic constriction not only improves RV function but also leads to less maladaptive RV remodeling in response to chronic RV pressure overload.
Progressive RV remodeling, including increased wall thickness and myocardial hypertrophy, as well as interstitial fibrosis, is a hallmark of PA hypertension. ${ }^{14}$ This initially adaptive hypertrophy eventually becomes maladaptive, and RV failure ensues. Clinically manifest RV failure is consistently reported as an adverse risk factor for survival in patients with pulmonary hypertension, ${ }^{4}$ and although its onset may be delayed by the use of advanced pulmonary vasodilator therapies, such as prostanoids, phosphodiesterase type 5 inhibitors, and endothelin receptor blockers, in the absence of a curative therapy it is likely that progression to RV failure and death will be the final common pathway for these patients. Although not ignoring the huge impact of vasodilator therapies, the potential for modifying RV function as a method of improving symptoms and survival has been emphasized in several recent reviews. ${ }^{15-17}$ Whereas these reviews have speculated that specific therapies may be developed to target the RV itself, there is evidence from both experimental and clinical studies that symptoms and outcomes may be driven by adverse ventricular-ventricular interactions related to the dilated and poorly functioning $\mathrm{RV}$ modifying the systolic and diastolic performance of the LV. We believe that the heart failure associated with progressive pulmonary hypertension should be considered a biventricular disease and that therapies targeted toward both ventricles may therefore be beneficial. Indeed, we and others have shown that other ventricular-ventricular interactions can be harnessed for potential therapeutic benefit, at least acutely, in the setting of pulmonary hypertension. ${ }^{11,12}$ For 

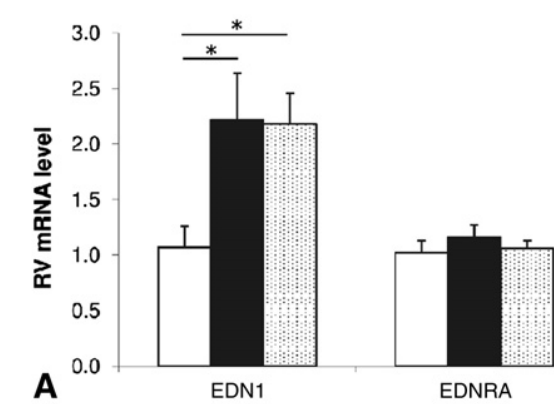

EDNRA
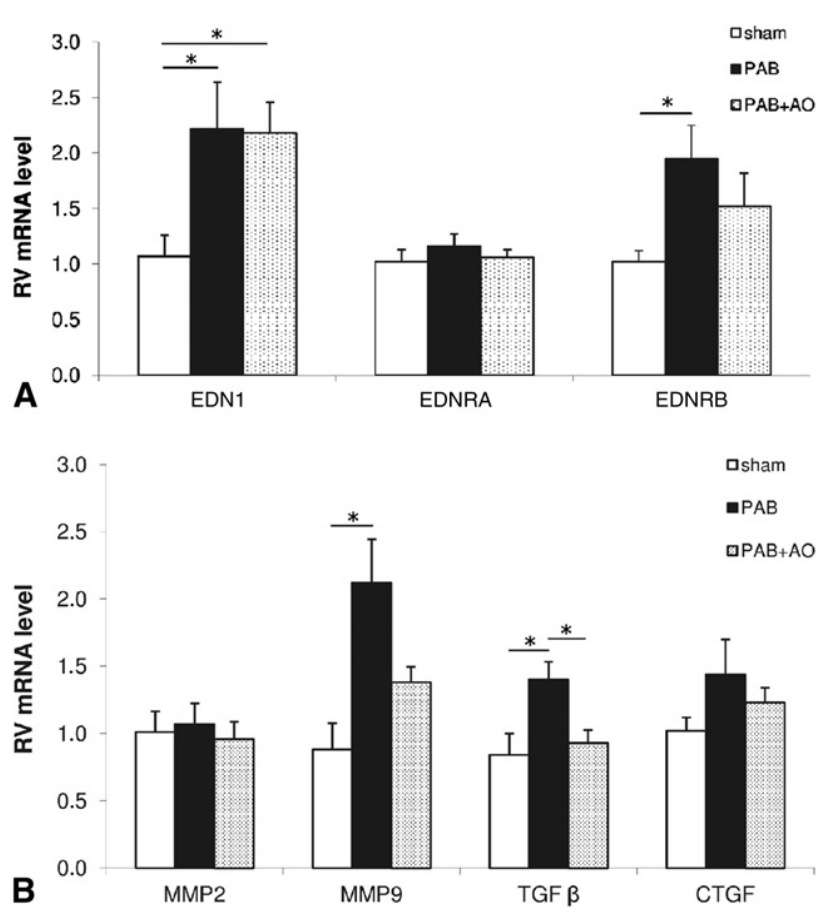

FIGURE 3. Results of real-time polymerase chain reaction analysis of right ventricular $(R V)$ myocardium. A, Expressions of endothelin 1 gene $(E D N 1)$ and endothelin receptor B gene (EDNRB) expressions were significantly increased in the pulmonary arterial banding $(P A B)$ group and in the group with added aortic banding $(P A B+A O)$ relative to the sham group, while there was no effect on endothelin receptor A gene (EDNRA) expression. B, Messenger RNA ( $m R N A$ ) levels for genes for matrix metalloproteinases $2(M M P 2)$ and $9(M M P 9)$, transforming growth factor- $\beta(T G F \beta)$ and connective tissue growth factor $(C T G F)$. Expressions of matrix metalloproteinase 9 and transforming growth factor $\beta$ genes were significantly increased in the pulmonary arterial banding group relative to the sham group. Addition of aortic banding was associated with significantly reduced transforming growth factor $\beta$ gene expression and a trend toward reduced matrix metalloproteinase 9 gene expression. There was no statistically significant change in connective tissue growth factor gene expression. Asterisk indicates $P<.05$.

example, increasing LV contractility through the Anrep effect (increased LV contractility in response to increased LV afterload by aortic constriction or pharmacologic systemic arterial vasoconstriction) leads to improved $\mathrm{RV}$ contractility in models of acute RV failure. To date, there have been no studies of this potential effect on biventricular responses to a chronically increased RV afterload, despite the potential therapeutic benefits that this novel approach may offer.

In the chronic PA banding model used in this study, RV remodeling was characterized by increases in both RV mass and cardiomyocyte diameter. In addition, there was excessive interstitial myocardial fibrosis, as evidenced by increased collagen content, characteristic of the failing RV myocardium. ${ }^{14}$ Concomitant aortic banding not only partially abrogated these histologic changes but also was
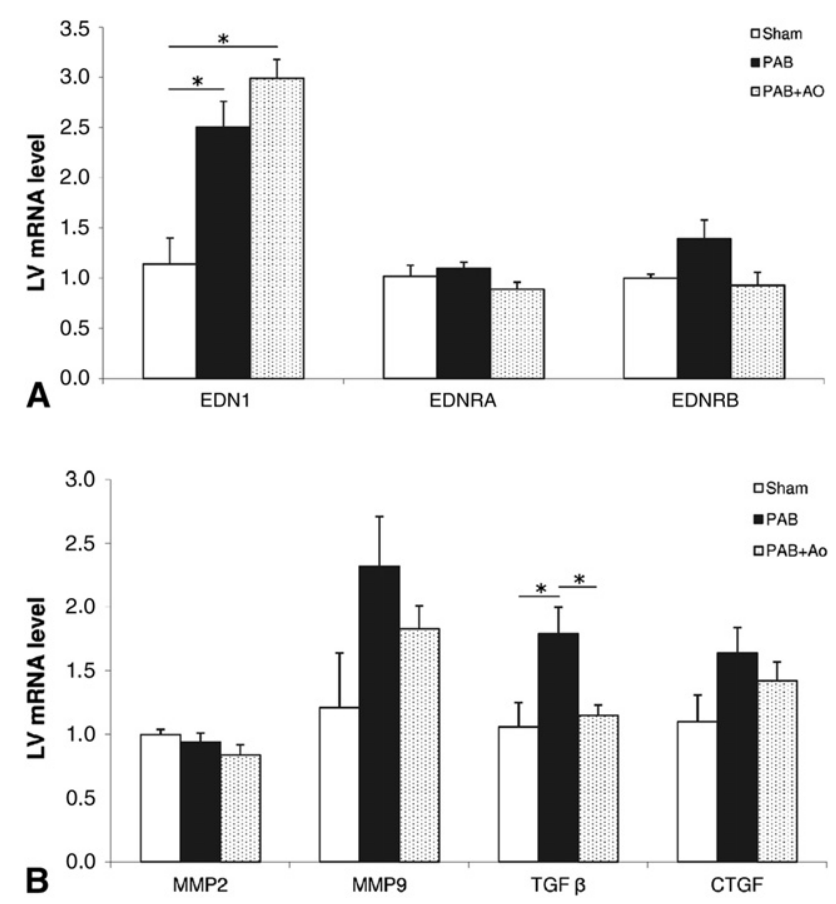

FIGURE 4. Results of real-time polymerase chain reaction analysis of left ventricular $(L V)$ myocardium. A, Expression of endothelin 1 gene (EDN1) was significantly increased in left ventricular myocardium in the pulmonary arterial banding $(P A B)$ group and in the group with added aortic banding $(P A B+A O)$ relative to the sham group, while there was no effect on endothelin receptor $\mathrm{A}$ gene $(E D N R A)$ and endothelin receptor $\mathrm{B}$ gene $(E D N R B)$ expressions. B, Messenger RNA ( $m R N A)$ levels for genes for matrix metalloproteinases 2 (MMP2) and 9 (MMP9), transforming growth factor $\beta$ (TGF $\beta$ ), and connective tissue growth factor $(C T G F)$. Transforming growth factor $\beta$ gene was significantly increased in the left ventricular myocardium in the pulmonary arterial banding group relative to the sham group. Addition of aortic banding was associated with significantly reduced transforming growth factor $\beta$ gene expression. There was no statistically significant change in matrix metalloproteinase 2 , matrix metalloproteinase 9, and connective tissue growth factor gene expressions in the left ventricular myocardium. Asterisk indicates $P<.05$.

associated with significantly improved RV function. Indeed, both the RV and the LV showed significantly improved systolic function, as measured by relatively load-independent indices derived from conductance catheter measurements. It is important to note that these improvements occurred in the presence of maladaptive RV remodeling (RV hypertrophy, increased cardiomyocyte diameter, and fibrosis) but before the development of overt RV failure (preserved RV ESPVR). When extrapolating to potential clinical applications, this seems to be the window when intervention would be most timely - when signs of RV remodeling are present, but before overt and irreversible RV failure has occurred.

The maladaptive hypertrophy seen in the PAB group was associated with marked changes in myocardial fibrotic signaling. The profile of increased $E D N 1, M M P 9$, and TGF- $\beta$ expressions observed in the PAB group is consistent with the 
maladaptive hypertrophy and fibrosis described in models of LV dysfunction and failure, and each presents potential therapeutic targets identified in a recent comprehensive review. ${ }^{5}$ Our data are consistent with those shown for the pressureloaded LV, and the effect of aortic banding is consistent with it being an intervention that alters the maladaptive signature, toward a more physiologic response. Concomitant aortic banding led to a decrease in histologic fibrosis and was associated with reduced TGF- $\beta, C T G F$, and $M M P 9$ expressions.

Although the signaling pathways that relate to these molecules have been studied extensively in the LV, less is known about their interrelationship in the RV subjected to chronic pressure load. TGF- $\beta$ regulates growth, differentiation, and function of different cell lineages (eg, myocytes, endothelial cells, and fibroblasts) and has recently been implicated in maladaptive RV failure associated with pulmonary hypertension. ${ }^{18}$ Furthermore, RV myocardial fibrosis, TGF- $\beta$ signaling, and degradation of the matrix by MMPs have shown to be linked in earlier studies of chronic pressure overload and to adversely affect myocardial systolic and diastolic function. ${ }^{19,20}$ In the LV, TGF- $\beta$ signaling regulates EDN1 expression via the JNK pathway, the effect of which in turn is regulated downstream by $C T G F$. Interestingly, the pattern of change in $C T G F$ expression in our groups (a rise in the PAB group, which was attenuated in the $\mathrm{PAB}+\mathrm{AO}$ group) was similar to those of TGF$\beta$ and $M M P 9$, although the magnitude of difference did not reach statistical significance. We did, however, observe significantly increased myocardial expression of $E D N 1$ in both the $\mathrm{PAB}$ and the $\mathrm{PAB}+\mathrm{AO}$ groups. Previous clinical studies of pulmonary hypertension have suggested that high circulating ET-1 levels are caused by increased gene expression and consequent synthesis, without a loss of the ET-1 clearance activity that normally occurs. $^{21-23}$ Whether our findings would be reflected by increased circulating levels of ET-1 or are limited to myocardial expression remains to be demonstrated, but it appears that the profibrotic responses to increased EDN1 expression (which was similarly elevated in both $\mathrm{PAB}$ and $\mathrm{PAB}+\mathrm{AO}$ groups, despite demonstrable differences in fibrosis by histologic examination) were in some way attenuated, presumably through regulation of upstream effects of reduced TGF- $\beta$ or downstream through reduced receptor stimulation. With regard to the latter, our findings of decreased $E D N R B$ expression may be important.

It was beyond the scope of this proof-of-principle study to explore fully the potential mechanisms by which aortic banding modifies the functional or molecular responses in the RV subjected to chronically increased afterload. A limitation of the study is that we are unable to comment on the mechanisms of adaptation with regard to the position of the ventricular septum. We speculate, however, that improved functional responses of the RV via the beneficial ventricular-ventricular interaction from increased LV afterload and increased contractility beneficially modifies the hemodynamic and myocardial milieu to reduce heart failure responses. Our finding of reduced MMP-9 signaling would be consistent with attenuation of myocardial oxidative stress, ${ }^{24}$ itself a potent stimulator of adverse remodeling. In turn, adverse proinflammatory and profibrotic responses are attenuated, leading to more physiologic hypertrophy of the myocardium.

The potential therapeutic implications of our findings must also remain similarly speculative. Systemic vasoconstrictor therapy to recapitulate the effects of aortic constriction in patients would be neither easy to perform nor readily adopted by clinicians and patients. Our observations regarding the profibrotic responses within the RV do raise the possibility of using other agents to modify RV responses. Endothelin blockade is already widely used for patients with advanced pulmonary hypertension, but it might be argued that it would need to be given earlier in the course of the disease if it were to have a beneficial effect on RV remodeling. In this regard, modification of TGF- $\beta$ signaling might be a more attractive target. It may be possible to modify fibrotic responses associated with increased TGF- $\beta$ signaling through angiotensin II with an angiotensin receptor blocker, in the same way as has been shown in models of LV disease. ${ }^{25}$

In summary, we have shown that the functional and molecular maladaptive responses to increased RV afterload are attenuated by concomitant aortic constriction, presumably through beneficial ventricular-ventricular interactions. The molecular responses suggest modification of profibrotic pathways, which in themselves may be therapeutic targets to improve RV remodeling in chronic pulmonary hypertension and other conditions of RV pressure afterload.

\section{References}

1. Berger RM, Beghetti M, Humpl T, et al. Clinical features of paediatric pulmonary hypertension: a registry study. Lancet. 2012;379:537-46.

2. Gaynor SL, Maniar HS, Bloch JB, Steendijk P, Moon MR. Right atrial and ventricular adaptation to chronic right ventricular pressure overload. Circulation. 2005;112(9 Suppl):I212-8.

3. Voelkel NF, Quaife RA, Leinwand LA, Barst RJ, McGoon MD, Meldrum DR, et al, National Heart, Lung, and Blood Institute Working Group on Cellular and Molecular Mechanisms of Right Heart Failure. Right ventricular function and failure: report of a National Heart, Lung, and Blood Institute working group on cellular and molecular mechanisms of right heart failure. Circulation. 2006; 114:1883-91.

4. Chin KM, Kim NH, Rubin LJ. The right ventricle in pulmonary hypertension. Coron Art Dis. 2005;16:13-8.

5. Leask A. Potential therapeutic targets for cardiac fibrosis: TGF $\beta$, angiotensin, endothelin, CCN2, and PDGF, partners in fibroblast activation. Circ Res. 2010; 106:1675-80.

6. Raymond RJ, Hinderliter AL, Willis PW, Ralph D, Caldwell EJ, Williams W, et al. Echocardiographic predictors of adverse outcomes in primary pulmonary hypertension. J Am Coll Cardiol. 2002;39:1214-9.

7. Gan CT, Lankhaar JW, Marcus JT, Westerhof N, Marques KM, Bronzwaer JG, et al. Impaired left ventricular filling due to right-to-left ventricular interaction in patients with pulmonary arterial hypertension. Am J Physiol Heart Circ Physiol. 2006;290:H1528-33.

8. Sanchez-Quintana D, Climent V, Ho SY, Anderson RH. Myoarchitecture and connective tissue in hearts with tricuspid atresia. Heart. 1999;81:182-91.

9. Damiano RJ Jr, La Follette P Jr, Cox JL, Lowe JE, Santamore WP. Significant left ventricular contribution to right ventricular systolic function. Am J Physiol. 1991;30(5 Pt 2):H1514-24. 
10. Yamashita H, Onodera S, Imamoto T, Obara A, Tanazawa S, Takashio T, et al. Functional and geometrical interference and interdependency between the right and left ventricle in cor pulmonale: an experimental study on simultaneous measurement of biventricular geometry of acute right ventricular pressure overload. Jpn Circ J. 1989;53:1237-44.

11. Belenkie I, Horne SG, Dani R, Smith ER, Tyberg JV. Effects of aortic constriction during experimental acute right ventricular pressure loading. Further insights into diastolic and systolic ventricular interaction. Circulation. 1995;92:546-54.

12. Apitz C, Honjo O, Friedberg MK, Assad RS, Van Arsdell G, Humpl T, et al. Beneficial effects of vasopressors on right ventricular function in experimental acute right ventricular failure in a rabbit model. Thorac Cardiovasc Surg. 2012;60:17-23.

13. Assad RS, Atik FA, Oliveira FS, Fonseca-Alaniz MH, Abduch MC, Silva GJ, et al. Reversible pulmonary trunk banding. VI: Glucose-6-phosphate dehydrogenase activity in rapid ventricular hypertrophy in young goats. $J$ Thorac Cardiovasc Surg. 2011;142(5):1108-13, 1113.e1.

14. Budev M, Arroliga A, Wiedemann H, Matthay RA. Cor pulmonale: an overview. Semin Respir Crit Care Med. 2003;24:233-44.

15. Handoko ML, de Man FS, Allaart CP, Paulus WJ, Westerhof N, VonkNoordegraaf A. Perspectives on novel therapeutic strategies for right heart failure in pulmonary arterial hypertension: lessons from the left heart. Eur Respir Rev. 2010;19:72-82

16. Banerjee D, Haddad F, Zamanian RT, Nagendran J. Right ventricular failure: a novel era of targeted therapy. Curr Heart Fail Rep. 2010;7:202-11.

17. Haddad F, Ashley E, Michelakis ED. New insights for the diagnosis and management of right ventricular failure, from molecular imaging to targeted right ventricular therapy. Curr Opin Cardiol. 2010;25:131-40.
18. Bogaard HJ, Natarajan R, Henderson SC, Long CS, Kraskauskas D, Smithson L, et al. Chronic pulmonary artery pressure elevation is insufficient to explain right heart failure. Circulation. 2009;120:1951-60

19. Janicki JS, Brower GL, Gardner JD, Forman MF, Stewart JA Jr, Murray DB, et al. Cardiac mast cell regulation of matrix metalloproteinase-related ventricular remodeling in chronic pressure or volume overload. Cardiovasc Res. 2006;69: 657-65.

20. Weber KT, Janicki JS, Shroff SG, Pick R, Chen RM, Bashey RI. Collagen remodeling of the pressure-overloaded, hypertrophied nonhuman primate myocardium. Circ Res. 1988;62:757-65.

21. Stewart DJ, Levy RD, Cernacek P, Langleben D. Increased plasma endothelin-1 in pulmonary hypertension: marker or mediator of disease? Ann Intern Med. 1991;114:464-9.

22. Giaid A, Yanagisawa M, Langleben D, Michel RP, Levy R, Shennib H, et al. Expression of endothelin-1 in the lungs of patients with pulmonary hypertension. $N$ Engl J Med. 1993;328:1732-9.

23. Langleben D, Dupuis J, Langleben I, Hirsch AM, Baron M, Senécal JL, et al. Etiology-specific endothelin-1 clearance in human precapillary pulmonary hypertension. Chest. 2006;129:689-95.

24. Awad AE, Kandalam V, Chakrabarti S, Wang X, Penninger JM, Davidge ST, et al Tumor necrosis factor induces matrix metalloproteinases in cardiomyocytes and cardiofibroblasts differentially via superoxide production in a PI3K $\gamma$-dependent manner. Am J Physiol Cell Physiol. 2010;298:C679-92.

25. Maejima Y, Okada H, Haraguchi G, Onai Y, Kosuge H, Suzuki J, et al. Telmisar$\tan$, a unique ARB, improves left ventricular remodeling of infracted heart by activating PPAR gamma. Lab Invest. 2011;91:932-44.

\section{COMMENTARY}

\section{Aortic constriction and the relevance of physiologic research}

\section{Emile A. Bacha, MD}

A casual reader might be tempted to skip over the article by Apitz and colleagues in this issue, dismissing it as irrelevant to clinical practice. The title is also somewhat offputting because "aortic constriction" evokes only bad images in the mind of any cardiac surgeon. In fact, however, this elegant yet traditional cardiac physiology experiment shows that chronic aortic constriction not only improves right ventricular (RV) function in the setting of chronic RV pressure overload but also leads to less maladaptive RV remodeling. This article does inform on highly relevant themes, such as ventricle-ventricle interaction, timing of RV remodeling, and the molecular signature of

\footnotetext{
From the Department of Cardiac Surgery, Morgan Stanley Children's Hospital of New York-Presbyterian, Columbia University, New York, NY.

Disclosures: Author has nothing to disclose with regard to commercial support.

Received for publication May 28, 2012; accepted for publication June 12, 2012; available ahead of print July 23, 2012

Address for reprints: Emile A. Bacha, MD, Columbia University, Department of Cardiac Surgery, Morgan Stanley Children's Hospital of New York-Presbyterian,

New York, NY 10032 (E-mail: eb2709@columbia.edu).

J Thorac Cardiovasc Surg 2012;144:1501

$0022-5223 / \$ 36.00$

Copyright (C) 2012 by The American Association for Thoracic Surgery

http://dx.doi.org/10.1016/j.jtcvs.2012.06.026
}

$\mathrm{RV}$ profibrotic reactions. It may also open the door to a very untraditional way of managing the remodeled RV. The problem of the hypertrophied yet not decompensated $\mathrm{RV}$ is typically solved by an early repair of the anatomy, such as early repair of tetralogy of Fallot for example. No one would consider "banding" the aorta to allow for reverse RV remodeling. What, however, about the thick, fibrotic RV in an older patient with unrepaired pulmonary stenosis? Should we consider, akin to pulmonary artery banding and left ventricular training in an older patient with transposition of the great arteries, a period of aortic afterload increase to reposition the septum and maybe reverse some of the myocardial profibrotic signaling? What about managing the failing postoperative RV? In the setting of good left ventricular function, and in the absence of any current drugs that can directly increase RV contractility, systemic afterload increase by pharmacologic means may in fact have a role to play.

At a time when therapeutic answers are being sought mostly in genetics and tissue engineering, this study reminds us that physiologic research remains an essential and vital tool. 